Artículo científico

Volumen 31(1):1-11. Enero-abril, 2020

e-ISSN 2215-3608, doi:10.15517/am.v31i1.37093

http://www.revistas.ucr.ac.cr/index.php/agromeso

\title{
Criterios de selección en líneas de tomate con hábito de crecimiento determinado $^{1}$
}

\section{Selection criteria in tomato lines with determinate growth habit}

\author{
Esteban Burbano-Erazo², Iván Javier Pastrana-Vargas², Julián Roberto Mejía-Salazar², \\ Franco Alirio Vallejo-Cabrera ${ }^{3}$
}

1 Recepción: 15 de julio, 2019. Aceptación: 16 de setiembre, 2019. Este trabajo fue elaborado a partir de los resultados de la tesis de maestría del primer autor, realizada en la Universidad Nacional de Colombia, Campus Palmira, Colombia.

2 Corporación Colombiana de Investigación Agropecuaria - AGROSAVIA. Centro de Investigación Motilonia - Km 5 Vía a Becerril - Agustín Codazzi, Cesar, Colombia. eburbanoe@unal.edu.co (autor para correspondencia, https://orcid.org/0000-0001-5056-9893); ipastrana@agrosavia.co; jmejia@agrosavia.co

3 Facultad de Ciencias Agropecuarias, Grupo de Investigación en Mejoramiento Genético, Agronomía y Producción de Semillas de Hortalizas, Universidad Nacional de Colombia, Palmira, Colombia. favallejoc@palmira.unal.edu.co

\section{Resumen}

Introducción. La selección de genotipos con hábito de crecimiento determinado en tomate, debe contemplar criterios adecuados de selección para incrementar la eficiencia del programa de fitomejoramiento. Objetivo. El objetivo de este trabajo fue estimar criterios de selección para líneas de tomate tipo "chonto" con hábito de crecimiento determinado. Materiales y métodos. Este trabajo se llevó a cabo en la Universidad Nacional de Colombia (Sede Palmira), en el año 2016, con siete líneas con hábito de crecimiento determinado y un testigo con crecimiento indeterminado. Se determinó la heredabilidad en sentido amplio $\left(\mathrm{h}^{2} \mathrm{~g}\right)$, coeficiente de variación ambiental, coeficiente de variación genética, eficiencia de la selección y ganancia genética, en parámetros morfológicos, fenológicos, de calidad de fruto, forma de fruto y producción, con el empleo del procedimiento RELM/BLUP, del software SELEGEN. Resultados. Hubo tres rangos de $\mathrm{h}^{2} \mathrm{~g}$, el primero con valores de $\mathrm{h}^{2} \mathrm{~g}$ mayores a 0,76 , el segundo entre 0,53 y 0,38 , y el tercero con un valor menor a 0,38 . Los mayores valores de $h^{2}$ g fueron para altura final de planta con 0,92 , altura de planta al momento de la cosecha con 0,88 , producción por planta con 0,83 , días a floración con 0,83 , número de frutos por planta con 0,82 , y días a cosecha con 0,82 . Para ganancia genética se encontró que el testigo presentó los mayores valores para, altura final de planta, altura de planta a cosecha, longitud de entrenudo, días a cosecha, duración de cosecha, contenido de sólidos solubles, número de frutos por planta, peso de fruto y producción por planta; sin embargo, en algunos parámetros como altura y fenología para selección por hábito de crecimiento determinado, resultaron mejores los valores más bajos. Conclusión. Se evidenciaron parámetros genéticos que podrían considerarse como criterios de selección para líneas de tomate tipo "chonto" con hábito de crecimiento determinado.

Palabras clave: fitomejoramiento, heredabilidad, parámetros genéticos, Solanum lycopersicum.
Abstract
Introduction. The selection of genotypes with a determined growth habit in tomato should contemplate adequate selection criteria to increase the efficiency of the plant breeding program. Objective. The objective of this 
research was to estimate selection criteria for "chonto" tomato lines with determined growth habit. Materials and methods. The research was developed in the Universidad Nacional de Colombia campus Palmira in 2016, using seven determinate habit growth lines (D) and a control with undetermined habit growth. The heritability in a broad sense $\left(h^{2} g\right)$, coefficient of environmental variation, coefficient of genetic variation, selection efficiency, and genetic gain was determined, in morphologic, phenological, quality fruit, fruit shape, and productions parameters using the RELM/BLUP process, from the SELEGEN software. Results. Three ranges of $\mathrm{h}^{2} \mathrm{~g}$ were found, the first with values of $\mathrm{h}^{2} \mathrm{~g}$ greater than 0.76 , the second between 0.53 and 0.38 , and the third with a value less than 0.38 . The highest values of $\mathrm{h}^{2} \mathrm{~g}$ were for final height plant with 0.92 , height plant to harvest with 0.88 , production per plant with 0.83 , days to flowering with 0.83 , fruit number with 0.82 , and days to harvest with 0.82 . For genetic gain it was found that the control presented the highest values within the main parameters for final height plant, plant height to harvest, internode length, days to harvest, crop duration, soluble solids content, number of fruits per plant, weight fruit, and plant production; however, in some parameters such as height and phenology for effects of selection by habit of determined growth lower values were better. Conclusion. Genetic parameters were evidenced that could be considered as selection criteria for "chonto" tomato lines with determined growth of habit.

Keywords: plant breeding, heritability, genetic parameters, Solanum lycopersicum.

\section{Introducción}

El cultivo de tomate (Solanum lycopersicum L.) se encuentra ampliamente distribuido en el mundo y representa uno de los alimentos de mayor consumo, debido a su participación tradicional en la dieta y su importante intervención en el fomento de la seguridad alimentaria (Hefferon, 2016). El aprovechamiento de la variabilidad genética de $S$. lycopersicum se origina de un fuerte proceso de premejoramiento, en el que la conservación, la caracterización y la identificación de genes promisorios para programas de mejoramiento genético representan los principales componentes (Nuez, 1995; Vallejo, 1999; de-Castro et al., 2010; Fernández-Moreno et al., 2017), lo que permite la identificación de caracteres de interés que confieren adaptación a condiciones bióticas y abióticas adversas (Saleem et al., 2016; Martínez et al., 2018). Para esto es necesario conocer los parámetros genéticos que contribuyen a la identificación de características que puedan ser heredables entre generaciones y que constituyen aspectos de interés en programas de mejoramiento genético (Holland et al., 2003; Resende, 2016).

En la actualidad, se han estimado parámetros genéticos dentro de investigaciones enfocadas a determinar el efecto del componente genético sobre la expresión de determinada característica (Haydar et al., 2007; Anjum et al., 2009; Mohamed et al., 2012; Ramzan et al., 2014; Khan et al., 2017), convirtiéndose en uno de los pasos fundamentales para la selección de genotipos promisorios por características de interés (Vallejo, 1999; Baena et al., 2003). La variación genética puede estar influenciada por efectos aditivos, no aditivos y de interacción no alélica; de esta manera parámetros como la heredabilidad $\left(\mathrm{h}^{2}\right)$ indican qué tanto de la expresión fenotípica está asociada al componente genético (Vallejo y Lobo, 1994).

La determinación de estos parámetros se puede realizar mediante diferentes metodologías; sin embargo, una de las más empleadas corresponde al análisis mediante el procedimiento RELM/BLUP (Resende, 2007). Este procedimiento corresponde a una metodología de modelo mixto de gran interés para la interpretación de resultados y posible identificación de características con potencial para selección de genotipos (Resende, 2016), además, dentro de un programa de mejoramiento resulta indispensable la estimación de componentes de varianza y valores genéticos (dos-Santos et al., 2016).

Un parámetro con alta heredabilidad y ganancia genética puede constituir un importante criterio de selección y un indicador del componente genético aditivo de la característica (Sharanappa y Mogali, 2014). 
El objetivo de este trabajo fue estimar criterios de selección para líneas de tomate tipo "chonto" con hábito de crecimiento determinado.

\section{Materiales y métodos}

\section{Sitio de estudio}

El estudio fue llevado a cabo en el Centro Experimental de la Universidad Nacional de Colombia, Campus Palmira (CEUNP), ubicado en el municipio de Candelaria, vereda el Carmelo a $3^{\circ} 25^{\prime} 34^{\prime \prime}$ latitud norte y $76^{\circ} 25^{\prime}$ 53" longitud oeste, a 951 msnm, departamento del Valle del Cauca, Colombia, durante el año 2016.

\section{Material vegetal}

El material genético correspondió a siete líneas de tomate tipo "chonto", caracterizado por presentar frutos "hombro verde", bi o tri loculares, peso promedio entre 70 y 100 g y maduración uniforme, principalmente (Vallejo, 1994), con hábito de crecimiento determinado (D2, D3, D5, D6, D7, D8 y D9), en cuarta generación de retrocruzamiento (RC4), más el testigo comercial (UNAPAL Maravilla) de crecimiento indeterminado, seleccionado por sus características deseables y amplia adopción por los productores; además, no se cuenta con cultivares tipo "chonto" de porte bajo, adaptados a la región. Las líneas se obtuvieron por el Grupo de Investigación en Mejoramiento Genético de Hortalizas de la Universidad Nacional, Sede Palmira, a partir del cruzamiento entre la variedad comercial UNAPAL Maravilla, también creada por la Universidad Nacional de Colombia, Sede Palmira y la variedad brasileña IPA-4, creada por el Instituto Pernambucano Agropecuario.

\section{Parámetros genéticos}

Con base en resultados generados en la investigación realizada por Burbano y Vallejo (2017), se estimaron parámetros genéticos para características i) morfológicas: altura final de planta (AFP), obtenida del promedio de altura de planta $(\mathrm{cm})$ de las seis plantas de la parcela útil al finalizar la cosecha; altura de planta al momento de la cosecha (APC), se tomó del promedio de altura de planta $(\mathrm{cm})$ de las seis plantas de la parcela útil al iniciar esta labor; diámetro de tallo (DT), correspondiente al promedio del diámetro del tallo principal (mm) de las seis plantas de la parcela útil; longitud de entrenudo (LE), promedio medido en el tallo principal ( $\mathrm{cm}$ ) de las seis plantas de la parcela útil, ii) fenológicas: días a floración (DAF), número de días cuando el $60 \%$ de las plantas de la parcela útil presentaron la primera flor abierta; días a cosecha (DAC), días cuando el $60 \%$ de las plantas de la parcela útil iniciaron el período de cosecha; duración de cosecha (DUC), diferencia entre el día de inicio y día final de la cosecha, iii) calidad y formato del fruto, contenido de sólidos solubles en grados Brix (GB), medido en cinco frutos

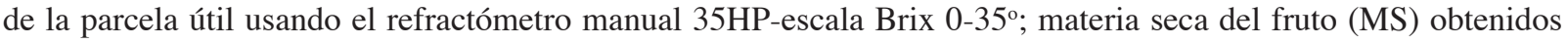
en porcentaje al extraer la diferencia de peso seco y peso fresco en cinco frutos de la parcela útil; diámetro de fruto (DF) medido en $\mathrm{mm}$, usando cinco frutos de la parcela útil; peso de fruto (PF), relación entre el peso total de frutos y el número de frutos cosechados por planta en la parcela útil, y iv) productivas: número de racimos por planta (NR), suma de todos los racimos producidos por planta dentro de la parcela útil; número de frutos por planta (NFP), suma de todos los frutos producidos por planta dentro de la parcela útil; y producción por planta (PP), producto del peso promedio de fruto $(\mathrm{kg})$ por el número de frutos por planta. 


\section{Análisis estadístico}

La información obtenida en esta investigación se analizó mediante el Sistema Estadístico de Selección Genética Computarizada SELEGEN REML/BLUP (Versión, 2014), el cual utiliza el procedimiento RELM/BLUP para estimación de varianzas (REML) y balanceo de datos (BLUP) (Resende, 2007). Se utilizó el modelo 20 (Ecuación 1), para la evaluación de genotipos (accesiones, cultivares, clones, híbridos, líneas y familias) en varias repeticiones y una observación por parcela (Resende, 2007), dado que el experimento se desarrolló bajo un diseño experimental en bloques completos al azar con tres repeticiones (Burbano y Vallejo, 2017) y en una sola localidad:

$$
\mathrm{y}=\mathrm{Xr}+\mathrm{Zg}+\mathrm{e} \quad(\text { ecuación } 1)
$$

Donde: "y" vector de datos, "Xr" vector de los efectos de repetición sumados a la media general, "Zg" vector de los efectos genotípicos y "e" vector del error o residuo.

Los parámetros estimados por el análisis correspondieron a: Vg: varianza genética, Ve: varianza ambiental, $\mathrm{h}^{2} \mathrm{~g}$ : heredabilidad en sentido amplio, $\mathrm{h}^{2} \mathrm{ml}$ : heredabilidad media lineal, AcL: eficiencia de la selección de líneas, CVgi\%: coeficiente de variación genética individual y CVe\%: coeficiente de variación asociado a los ambientes.

\section{Resultados}

El coeficiente de variación asociado al ambiente (CVe\%) varió entre 2,29 y 18,79 \%; estos valores son considerados bajos y muestran la importancia del componente genético en la expresión de los caracteres evaluados. El valor más alto se presentó en el parámetro MS y el más bajo en PF. El valor más bajo del coeficiente de variación genética $(\mathrm{CVg} \%)$ fue para PF y el más alto para APC, con valores de $0,55 \%$ y $21,16 \%$, respectivamente (Cuadro 1). Caracteres con mayor variación genética individual ofrecen mayor posibilidad de selección en el grupo de individuos evaluados.

Los valores de $\mathrm{h}^{2} \mathrm{~g}$ encontrados fueron altamente contrastantes, entre 0,002 y 0,92 , para las variables DT y AFP, respectivamente. Con los valores de $\mathrm{h}^{2} \mathrm{~g}$ obtenidos, se separaron las variables en tres grupos, el primero con valores de $\mathrm{h}^{2}$ g mayores a 0,76 , en el que estuvieron los parámetros AFP, APC, PP, DF, NFP, DAC y LE, en orden decreciente. El segundo grupo, con valores entre 0,53 y 0,38, para NR, PF, DF, GB, DUC y MS; y el tercero, con valores menores a 0,38 , en el que solamente estuvo el parámetro DT (Cuadro 1). En cuanto a la eficiencia de selección para los parámetros medidos se encontró que los valores oscilaron entre 0,09 y 0,99 , siendo los valores mayores de 0,95 los estimados por las variables de respuesta AFP, APC, LE, DAF, DAC, NFP, PP; mientras que el menor valor correspondió a DT.

En cuanto al porcentaje de ganancia genética (GG) (Cuadro 2), se encontró que el testigo comercial presentó los valores más altos en todas las variables de respuesta, respecto al promedio de la población evaluada, a excepción de los resultados estimados en los parámetros DF y NR, en los que la GG fue mayor para las líneas de crecimiento determinado. Uno de los parámetros de mayor interés en la selección de materiales con porte bajo de planta es la AFP, en la que se encontró la mayor GG en el testigo con $47 \%$, mientras que las líneas D5, D2, D6 y D3 tuvieron $-16 \%,-13 \%,-12 \%,-12 \%$, respectivamente, esto indica que estas líneas presentaron menor altura respecto al promedio de la población. Lo que, en el enfoque de esta investigación, resulta favorable, ya que la selección va dirigida hacia los genotipos de menor altura, para reducir costos de producción. En la GG de APC, se encontró el mismo comportamiento que en AFP. 
Cuadro 1. Estimación de parámetros genéticos y residuales de variables de respuesta morfológicas, fenológicas y productivas en líneas de tomate tipo "chonto" (Solanum lycopersicum L.), en Palmira, Valle del Cauca, Colombia. 2016.

Table 1. Estimation of genetic and residual parameters of morphological, phenological, and productive response variables in "chonto" tomato (Solanum lycopersicum L.) lines, in Palmira, Valle del Cauca, Colombia. 2016.

\begin{tabular}{ccccccccc}
\hline Variable & $\begin{array}{c}\text { Varianza } \\
\text { genética }\end{array}$ & $\begin{array}{c}\text { Varianza } \\
\text { ambiental }\end{array}$ & $\begin{array}{c}\text { Varianza } \\
\text { fenotípica }\end{array}$ & $\begin{array}{c}\text { Heredabili- } \\
\text { dad }\end{array}$ & $\begin{array}{c}\text { Heredabili- } \\
\text { dad media } \\
\text { lineal }\end{array}$ & $\begin{array}{c}\text { Eficiencia } \\
\text { de la } \\
\text { selección }\end{array}$ & $\begin{array}{c}\text { Coeficiente } \\
\text { de variación } \\
\text { genético }\end{array}$ & $\begin{array}{c}\text { Coeficiente } \\
\text { de variación } \\
\text { ambiental }\end{array}$ \\
\hline AFP & 730,22 & 83,87 & 814,08 & $0,92 \pm 0,47$ & 0,97 & 0,99 & 20,64 & 6,99 \\
APC & 597,36 & 83,76 & 681,12 & $0,88 \pm 0,46$ & 0,97 & 0,98 & 21,16 & 7,92 \\
LE & 1,79 & 0,58 & 2,37 & $0,76 \pm 0,43$ & 0,93 & 0,96 & 13,72 & 7,78 \\
DT & 0,008 & 3,69 & 3,7 & $0,002 \pm 0,02$ & 0,009 & 0,09 & 0,55 & 11,3 \\
DAF & 5,68 & 1,13 & 6,81 & $0,83 \pm 0,45$ & 0,95 & 0,98 & 11,54 & 5,14 \\
DAC & 17,48 & 3,91 & 21,39 & $0,82 \pm 0,45$ & 0,95 & 0,97 & 5,58 & 2,64 \\
DUC & 4,67 & 7,33 & 12 & $0,39 \pm 0,31$ & 0,72 & 0,85 & 13,42 & 16,82 \\
GB & 0,05 & 0,06 & 0,11 & $0,42 \pm 0,32$ & 0,74 & 0,86 & 5,06 & 5,97 \\
MS & 0,53 & 0,87 & 1,4 & $0,38 \pm 0,3$ & 0,71 & 0,84 & 14,71 & 18,79 \\
DF & 0,0005 & 0,0007 & 0,001 & $0,42 \pm 0,32$ & 0,74 & 0,86 & 2,12 & 2,51 \\
NR & 2,22 & 1,93 & 4,14 & $0,53 \pm 0,36$ & 0,82 & 0,91 & 11,16 & 10,41 \\
NFP & 42,48 & 10,65 & 53,13 & $0,82 \pm 0,44$ & 0,94 & 0,97 & 18,18 & 9,1 \\
PF & 4,18 & 5,36 & 9,54 & $0,44 \pm 0,33$ & 0,76 & 0,87 & 2,02 & 2,29 \\
PP & 511648,6 & 104691,8 & 616340,5 & $0,83 \pm 0,45$ & 0,95 & 0,98 & 19,56 & 8,85 \\
\hline
\end{tabular}

Altura final de planta (AFP), altura de planta al momento de la cosecha (APC), diámetro de tallo (DT), longitud de entrenudo (LE), días a floración (DAF), días a cosecha (DAC), duración de cosecha (DUC), contenido de sólidos solubles en grados Brix (GB), materia seca del fruto (MS), diámetro de fruto (DF), peso de fruto $(\mathrm{PF})$, número de racimos por planta (NR), número de frutos por planta (NFP), y producción por planta (PP) / Final height plant (AFP), height plant to harvest (APC), stalk diameter (DT), internode length (LE), days to flowering (DAF), days to harvest (DAC), harvest duration (DUC), content of soluble solids in Brix degrees (GB), fruit dry matter $(\mathrm{MS})$, fruit diameter (DF), fruit weight (PF), bunches per plant (NR), fruit number per plant (NFP), and plant production (PP).

El parámetro DT presentó la menor ganancia genética (GG), esto debido probablemente a que es una variable afectada por condiciones ambientales y a que la variación entre genotipos de diferente hábito de crecimiento no fue contrastante. Mientras que para LE, el testigo presentó la mayor GG con 32 \%, en comparación con la media de toda la población evaluada.

Al analizar las variables fenológicas, el periodo necesario para llegar a floración resulta de gran interés, debido a que es una característica que condiciona la precocidad de las especies para reproducirse, en este caso para avanzar en el proceso de fructificación. En esta investigación se encontró que el testigo presentó la mayor GG con $19 \%$, siendo el material genético más tardío para florecer, mientras que las líneas D5, D2, D3 y D6, tuvieron valores negativos, indicando su mayor precocidad respecto a la población con -19\%, -9 \%, -2 \% y -2 \%, respectivamente. Para DAC, en la que los valores de ganancia genética fueron bajos, el testigo presentó el mayor valor con $9 \%$, siendo el genotipo más tardío, mientras que el menor \%GG fue obtenido por las mismas líneas de crecimiento determinado mencionadas anteriormente. En este parámetro fenológico resulta de gran interés una mayor precocidad. En DUC se encontró una GG baja en las líneas evaluadas, respecto al testigo que presentó el valor más alto con $17 \%$. Este parámetro resulta de interés, debido a que permite concentrar la labor de cosecha, incrementando la eficiencia y evitando posibles pérdidas por condiciones bióticas y abióticas.

Para los parámetros de calidad y forma de fruto se encontró que, en GB el testigo presentó el valor más alto de GG (10\%), superando a las líneas de hábito de crecimiento determinado; mientras que para la materia seca del fruto 
Cuadro 2. Ganancia genética de parámetros genéticos y residuales de variables de respuesta morfológicas, fenológicas y productivas en líneas de tomate tipo "chonto" (Solanum lycopersicum L.), en Palmira, Valle del Cauca, Colombia. 2016.

Table 2. Genetic gain of genetic and residual parameters of morphological, phenological and productive response variables in "chonto" tomato (Solanum lycopersicum L.) lines, in Palmira, Valle del Cauca, Colombia. 2016.

\begin{tabular}{ccccccccccccccc}
\hline Línea & AFP & APC & DT & LE & DAF & DAC & DUC & GB & MS & DF & NR & NFP & PF & PP \\
\hline D2 & $-12,73$ & $-15,32$ & $-0,051$ & $-8,15$ & $-8,79$ & $-2,80$ & 1,81 & 1,10 & 15,56 & 0,10 & 15,29 & 1,57 & $-2,59$ & $-2,46$ \\
D3 & $-12,08$ & $-11,79$ & $-0,008$ & $-4,59$ & $-1,87$ & $-5,01$ & 5,16 & $-1,10$ & $-6,26$ & $-1,35$ & $-0,40$ & 10,11 & $-1,69$ & 7,25 \\
D5 & $-16,02$ & $-15,03$ & $-0,032$ & $-5,30$ & $-19,17$ & $-5,64$ & 7,39 & $-1,10$ & 11,99 & $-1,10$ & 8,37 & 12,94 & $-0,20$ & 12,12 \\
D6 & $-12,21$ & $-13,65$ & $-0,001$ & $-4,12$ & $-1,87$ & $-5,01$ & 5,16 & $-1,54$ & $-0,89$ & $-0,77$ & 10,22 & 12,39 & $-1,32$ & 9,04 \\
D7 & 1,29 & 3,63 & 0,051 & $-2,46$ & 7,35 & 4,46 & $-17,15$ & $-2,42$ & $-10,91$ & 2,69 & $-10,41$ & $-23,25$ & 0,31 & $-23,80$ \\
D8 & 1,90 & 4,76 & $-0,024$ & $-4,83$ & 0,43 & 2,57 & $-9,34$ & $-2,86$ & $-19,86$ & 1,39 & $-9,02$ & $-20,07$ & 1,62 & $-19,48$ \\
D9 & 3,27 & 0,41 & 0,006 & $-2,93$ & 5,04 & 2,25 & $-10,46$ & $-2,42$ & $-1,25$ & 1,78 & $-10,10$ & $-16,68$ & 1,75 & $-15,46$ \\
T & 46,59 & 46,98 & 0,058 & 32,39 & 18,88 & 9,20 & 17,43 & 10,35 & 11,63 & $-2,73$ & $-3,94$ & 23,00 & 2,12 & 32,80 \\
\hline
\end{tabular}

Altura final de planta (AFP), altura de planta al momento de la cosecha (APC), diámetro de tallo (DT), longitud de entrenudo (LE), días a floración (DAF), días a cosecha (DAC), duración de cosecha (DUC), contenido de sólidos solubles en grados Brix (GB), materia seca del fruto (MS), diámetro de fruto (DF), peso de fruto (PF), y número de racimos por planta (NR), número de frutos por planta (NFP), y producción por planta (PP) / Final height plant (FHP), height plant to harvest (HPH), stalk diameter (DT), internode length (LE), days to flowering (DAF), days to harvest (DAC), harvest duration (DUC), content of soluble solids in Brix degrees (GB), fruit dry matter (MS), fruit diameter (DF), fruit weight (PF), bunches per plant (NR), fruit number per plant (NFP), and plant production (PP).

(MS) el mayor valor fue obtenido por la línea D2 (16\%), seguida de la línea D5 (12\%) que tuvo el mismo resultado que el testigo. El diámetro de fruto no presentó valores contrastantes, debido a que todos los materiales genéticos utilizados fueron líneas con formato de fruto tipo "chonto". Para PF se encontró que las líneas de crecimiento determinado D8, D9 y el testigo presentaron una GG baja del $2 \%$.

En cuanto a los componentes de rendimiento, el número de racimos (NR) presentó un comportamiento de interés para GG, debido a que D2 (15\%), D6 (10 \%) y D5 (8 \%) superaron al testigo (-4 \%), esto posiblemente se debió a que las plantas de porte bajo presentaron una cosecha concentrada y pudieron expresar su potencial productivo en un solo periodo, mientras que el hábito indeterminado continua su proceso de producción de racimos de manera prolongada. En NFP se encontró que la mayor GG fue para el testigo con $23 \%$, seguido de las líneas D2, D6 y D5, con $15 \%, 10 \%$ y 8\%, respectivamente. Finalmente, en cuanto a la producción por planta, se encontró que las plantas de mayor altura, representadas por el testigo, alcanzaron la mayor ganancia genética, seguido de las líneas D5 y D6, con $33 \%, 12 \%$ y $7 \%$, respectivamente.

\section{Discusión}

Se encontraron resultados de interés en cuanto $\mathrm{a} \mathrm{h}^{2} \mathrm{~g}$, ya que se sugiere que valores mayores a $20 \%$ son importantes como criterios de selección (Resende, 2002) y dentro de este rango estuvieron todas las variables de respuesta con la única excepción de DT, que correspondió al valor más bajo. Además, valores altos de h²g y GG también se relacionaron con una alta eficiencia de selección (Falconer y Mackay 1996; Kumar et al., 2013). Es así que la selección de estas líneas, con hábito de crecimiento determinado, puede ser apoyada por el uso de otros criterios morfológicos, fenológicos, de calidad y formato de fruto y productivos (Vallejo, 1999; Burbano y Vallejo, 2017). 
La determinación de parámetros genéticos resulta de gran interés para la selección de genotipos en programas de mejoramiento genético (Resende, 2016). En cuanto a los parámetros del componente morfológico, como DT, se encontró la más baja $\mathrm{h}^{2} \mathrm{~g}$ con 0,002 , debido probablemente a que es una característica influenciada por el ambiente (Wray y Visscher, 2008; De-Swaef y Steppe, 2010), una respuesta similar para este carácter fue reportada por Peralta et al. (2012) en líneas avanzadas de tomate. Otro parámetro morfológico cuantificado fue la LE, característica que evidenció un alto $\% \mathrm{CVg}$, debido probablemente a las diferencias existentes entre plantas con hábitos de crecimiento contrastantes, además, esta característica es un atributo diferencial, desde el punto de vista genético, siendo los entrenudos más cortos en plantas de porte bajo o hábito de crecimiento determinado (Nuez, 1995).

La altura de la planta (AFP y APC) se considera un parámetro de gran interés en programas de mejoramiento de tomate (Kumar et al., 2013), y puede estar afectada por factores de tipo genético y ambiental (Vallejo, 1999). Si se tiene en cuenta el hábito de crecimiento como determinante de la altura de planta, se reporta que esta condición está gobernada, principalmente, por un gen recesivo en estado homocigoto (self pruning, spsp) para el hábito de crecimiento determinado (Pnueli et al., 1998; Vallejo, 1999; Carmel-Goren et al., 2003; Finzi et al., 2017). En este estudio los mayores valores de $\mathrm{h}^{2} \mathrm{~g}$ se determinaron para AFP 0,92 y para APC 0,88 , que, a su vez, permitieron determinar altas GG que podrían relacionar estos parámetros con genes de tipo aditivo (Kumar et al., 2013), por tanto, podría considerarse que la selección por hábito de crecimiento es factible teniendo en cuenta como indicador la altura de planta. En híbridos de tomate se han determinado valores de 0,90 de $\mathrm{h}^{2} \mathrm{~g}$ (Mérida et al., 2017), entretanto, Kumar et al. (2013) reportaron un valor de GG de 63,36\% para altura de planta en genotipos de tomate. El \%CVg, obtenido en estos parámetros, fue de los más altos al compararse con el resultado de las otras variables de respuesta esto puede deberse a que dentro de la población evaluada se encontraban genotipos con hábito de crecimiento determinado e indeterminado. Por otro lado, también es válido mencionar que la altura de planta sin considerar el tipo de hábito de crecimiento, se ha reportado como un parámetro o descriptor cuantitativo, que puede tener una $\mathrm{h}^{2} \mathrm{~g}$ mayor a 0,50 (Baena et al., 2003).

El periodo necesario para iniciar floración (DAF) presentó un \% CVg bajo $(11,54)$, y un valor alto para $\mathrm{h}^{2} \mathrm{~g}$ $(0,83)$, relacionado con una alta eficiencia de selección estimada, lo que sugiere que podría considerarse promisorio como criterio de selección. La $\mathrm{h}^{2} \mathrm{~g}$ obtenida fue menor pero comparable con lo encontrado en una evaluación de híbridos de tomate, en la que se encontraron valores de 0,96 (Shokat et al., 2015; Mérida et al., 2017), no obstante, Nuez (1995) mencionó que la precocidad en el periodo de floración es mayor en cultivares con hábito de crecimiento determinado, lo que también ha sido sustentado por Burbano y Vallejo (2017). Para DAF se han reportado valores de GG del 19,8 \% (Shokat et al. 2015) y 19,9 \% (Haydar et al., 2007), en evaluación de híbridos de tomate y en materiales genéticos provenientes de una colección de germoplasma, respectivamente, valores similares al registrado por el testigo en este estudio.

El parámetro días a cosecha (DAC) presentó un $\% \mathrm{CVg}$ de 5,58, siendo un valor bajo, lo que permite inferir que las líneas no variaron mucho en cuanto a esta característica y que el efecto ambiental fue reducido en este rasgo, lo que permitió una estimación fiable de $\mathrm{h}^{2} \mathrm{~g}(0,82)$ que sugiere que es una característica asociada al componente genético. En general, los reportes de $\mathrm{h}^{2}$ son altos para este parámetro, del orden de 0,98 (Shokat et al., 2015) y 0,96 (Mérida et al., 2017), estimados a partir de la evaluación de híbridos de tomate. Entretanto, la duración de cosecha (DUC), parámetro considerado de interés, dependiendo del enfoque y tipo de cultivo, en el caso de cultivos tipo industrial, una corta duración de la cosecha incrementa la eficiencia de la labor y posiblemente reduce costos de producción por la reducción de gastos en materiales, insumos y mano de obra. De esta manera podría considerarse un criterio de selección, sin embargo, en esta investigación el valor fue bajo para $\mathrm{h}^{2} \mathrm{~g}$, lo cual no concuerda con otras evaluaciones en las que se obtuvo un valor de 0,76 , indicando una buena participación del componente genético en este parámetro (Kumar et al., 2013), no obstante, la h²ml dada por la selección particular de líneas sí se registra en 0,72, la cual es alta. Nuez (1995) mencionó que la duración corta puede ser un rasgo de los materiales genéticos de hábito de crecimiento determinado. La DUC es una característica que se relaciona con el tipo de planta, debido 
a que cultivares con hábito de crecimiento determinado se caracterizan por presentar un periodo de cosecha corto (Filgueira, 2000), en razón a esto, la selección se enfocaría en las líneas con menor GG (Vallejo, 1999). Algunos autores reportaron GG del 5,20 \% para el periodo de formación de frutos (Shokat et al., 2015), lo que indica que este parámetro también ha presentado valores bajos en otras investigaciones.

En general, la selección del testigo marca una mayor GG en la mayoría de los parámetros estimados, mientras que para las líneas, en su mayoría, presentaron valores negativos de GG, lo que resulta coherente al tener en cuenta las diferencias contrastantes entre cultivares con hábito de crecimiento indeterminado y determinado (Nuez, 1995; Vallejo, 1999; Burbano y Vallejo, 2017). GG similar se encontró en otra investigación donde el valor fue de 25,29 para DAC y 17,79 \% para DUC (Kumar et al., 2013). Una inferencia preliminar permite catalogar a estas variables fenológicas como rasgos de interés en la selección de materiales genéticos mejorados.

El contenido de sólidos solubles GB, presentó un valor intermedio de $\mathrm{h}^{2} \mathrm{~g}$ con 0,42 , lo que indica una interesante influencia genética en esta investigación, a pesar de que esta característica pueda estar afectada por múltiples condiciones fisiológicas y ambientales (Yelle et al., 1991), así como características de crecimiento, dado que cultivares con crecimiento indeterminado pueden presentar mayores valores de GB que plantas de porte bajo (Kolota y Adamczewska-Sowinska, 2001). Para este parámetro se han determinado valores de $\mathrm{h}^{2} \mathrm{~g}$ de 0,55 (López et al., 2015) hasta 76,29 \%, con GG bajas (Kumar et al., 2013). Por otra parte, la MS presentó una baja $\mathrm{h}^{2} \mathrm{~g}$ y un alto \%CVe, que sugiere influencia de factores ambientales, sin embargo, en cuanto GG las líneas D2 (16 \%) y D5 (12\%) fueron mayores e iguales al testigo (12\%), lo que resulta favorable en la selección de las líneas con hábito de crecimiento determinado.

Los parámetros DF y PF son caracteres importantes para diferenciar el tipo de tomate, el último en un rango de peso para frutos tomates tipo "chonto" entre 80 y $180 \mathrm{~g}$ (Burbano y Vallejo, 2017) y ambos representan variables de interés dentro de los componentes de rendimiento. Para el parámetro DF existe una marcada influencia ambiental, determinándose una $\mathrm{h}^{2} \mathrm{~g}$ intermedia $(0,42)$, que se encuentra entre valores reportados por otros autores para este parámetro genético desde 0,23 hasta 0,66 (Peralta et al., 2012; López et al., 2015). Entretanto, el PF presentó un $\% \mathrm{CVg}$ de 2,02, debido probablemente a que los materiales genéticos evaluados comparten información genética (Burbano y Vallejo, 2017) y su formato de fruto se encuentra dentro del rango establecido para el fruto tipo "chonto". $\mathrm{La} \mathrm{h}^{2} \mathrm{~g}$ fue intermedia, indicando que este carácter puede estar influenciado por el componente genético y ambiental u otros efectos de interacción entre genes. El efecto ambiental sobre este parámetro también fue reportado en la investigación de Ahmad et al. (2017), quienes encontraron un valor de 0,24 para la h ${ }^{2} \mathrm{~g}$ de este carácter, indicando una baja influencia del componente genético sobre esta característica. Falconer y Mackay (1996) sugirieron que características con dichos niveles de $\mathrm{h}^{2} \mathrm{~g}$ pueden estar relacionadas con efectos poligénicos.

La producción por planta (PP) correspondió a uno de los parámetros de mayor interés para mejorar. En este análisis se encontró que el \%CVg fue uno de los más altos $(8,85 \%)$ al compararse con el valor de los otros caracteres medidos en esta investigación, esto se puede deber probablemente a que este es un carácter poligénico, además que se evaluaron líneas de diferente hábito de crecimiento. $\mathrm{La} \mathrm{h}^{2} \mathrm{~g}$ fue alta $(0,83 \%)$, indicando una alta influencia genética sobre este parámetro, acorde a lo reportado en híbridos de tomate con valores de $\mathrm{h}^{2} \mathrm{~g}$ de 0,87 (Vallejo y Lobo, 1994; Mérida et al., 2017), sin embargo, los mismos autores mencionaron la dificultad para mejorar en cuanto a este parámetro, debido a la influencia de otros factores, tal como la asociación inversa entre número y peso de frutos. En cuanto a la GG de las líneas con crecimiento determinado, se encontraron valores más bajos que el testigo, tal como se ha reportado al comparar cultivares con diferentes hábitos de crecimiento (Nuez, 1995; Vallejo, 1999); sin embargo, es necesario analizar los costos de producción en los dos tipos de planta, para conocer la relación costo-beneficio.

El NFP presentó una $\mathrm{h}^{2} \mathrm{~g}$ alta $(0,82)$, que se considera altamente heredable de acuerdo con la evaluación; sin embargo, es menor a lo reportado por Vallejo y Lobo (1994) y Ramzan et al. (2014) en híbridos de tomate, con valores de 0,93 y 1,00 , respectivamente. Este parámetro productivo presentó una GG intermedia para el testigo, 
seguida de las líneas D5, D6 y D3, lo que destaca la participación de plantas con menor altura. Este resultado difiere del 92,11\% encontrado en otra investigación (Kumar et al., 2013), sin embargo, esto depende del tipo de población evaluada.

Finalmente, para el número de racimos por planta (NR) se encontró una $\mathrm{h}^{2} \mathrm{~g}$ intermedia, cuyo valor fue más bajo que lo reportado para híbridos de tomate $(0,94)$ (Ramzan et al., 2014), no obstante, se debe considerar que en el presente trabajo las estimaciones se basaron en evaluación de líneas. Se estimó un valor intermedio de \%CVg $(11,6 \%)$, mostrando que se presentó una variación dentro de las líneas de tomate tipo "chonto" con crecimiento determinado y el testigo indeterminado. Algunos autores reportaron una GG de 3,17\% para la cantidad de frutos por racimos (Shokat et al., 2015), mientras que en otro reporte se menciona un valor alto del $80 \%$ de GG, lo que pudo deberse a que el comportamiento productivo fue muy contrastante al provenir de una colección de germoplasma (Haydar et al., 2007), a diferencia de esta investigación en la que las líneas fueron avanzadas y homogéneas. Esto permitió evidenciar el importante potencial productivo de estas líneas, ya que, a pesar de su menor producción, pueden presentar beneficios en cuanto a menor requerimiento de mano de obra, tutorado, materiales e insumos agropecuarios, que se agrupan dentro de los costos de producción, tal como se ha mencionado en otras investigaciones (Vallejo 1999; Burbano y Vallejo, 2017).

\section{Conclusión}

Los criterios de selección basados en parámetros morfológicos, fenológicos y productivos, como altura de planta, longitud de entrenudo, días a floración, días a cosecha, número de frutos por planta y producción de planta, pueden resultar de interés para la selección de genotipos de tomate con hábito de crecimiento determinado, debido a su alta $\mathrm{h}^{2} \mathrm{~g}$. Además, en las líneas de tomate que se utilizaron en esta investigación, la ganancia genética negativa para la altura de planta constituyó un indicador favorable para la obtención de plantas de porte bajo, tal como se evidenciaron en las líneas D2, D3, D5 y D6, a pesar de que se haya presentado una menor ganancia genética en producción por planta.

\section{Literatura citada}

Ahmad, M., M. Iqbal, B.A. Khan, Z.U. Khan, K. Akbar, I. Ullah, S. Muhammad, and A. Rehman. 2017. Response to selection and decline in variability, heritabilty and genetic advance from F2 to F3 generation of tomato (Solanum lycopercicum). Int. J. Plant Res. 7:1-4. doi:10.5923/j.plant.20170701.01

Anjum, A., N. Raj, A. Nazeer, and S.H. Khan. 2009. Genetic variability and selection parameters for yield and quality attributes in tomato. Ind. J. Hortic. 66:73-78.

Baena, D., F.A. Cabrera, y E.I. Salazar. 2003. Avance generacional y selección de líneas promisorias de tomate (Lycopersicon esculentum Mill) tipos chonto y milano. Acta Agron. 52(1):1-9.

Burbano, E., y F.A. Vallejo. 2017. Producción de líneas de tomate "chonto”, Solanum lycopersicum Mill., con expresión del gen sp responsable del crecimiento determinado. Rev. Colomb. Cienc. Hort. 11:63-71. doi:10.17584/rcch.2017v11i1.5786

Carmel-Goren, L., Y.S. Liu, E. Lifschitz, and D. Zamir. 2003. The Self-Pruning gene family in tomato. Plant Mol. Biol. 52:12151222. doi:10.1023/B:PLAN.0000004333.96451.11

de-Castro, J.P.A., C. Nick, C.C. Milagres, A.P. Mattedi, B.G. Marim, and D.J.H. da-Silva. 2010. Genetic diversity among tomato's subsamples for pre-breeding. Crop Breed. Appl. Biotechnol. 10:74-82. doi:10.12702/1984-7033.v10n01a10 
De-Swaef, T., and K. Steppe. 2010. Linking stem diameter variations to sap flow, turgor and water potential in tomato. Funct. Plant Biol. 37:429-438. doi:10.1071/FP09233

dos-Santos, A., G. Ceccon, P.E. Teodoro, A.M. Correa, R.D.C.F. Álvarez, J.F. da-Silva, e V.B. Alves. 2016. Adaptabilidade e estabilidade de genótipos de feijão caupi ereto via REML/BLUP e GGE Biplot. Bragantia 75:299-306. doi:10.1590/16784499.280

Falconer, D.S., and F.C.T. Mackay. 1996. Introduction to quantitative genetic. $4^{\text {th }}$ ed. Longman Group Limited, Harlow, Essex, GBR.

Fernández-Moreno, J.P., D. Levy-Samoha, S. Malitsky, A.J. Monforte, D. Orzaez, A. Aharoni, and A. Granell. 2017. Uncovering tomato quantitative trait loci and candidate genes for fruit cuticular lipid composition using the Solanum pennellii introgression line population. J. Exp. Bot. 68:2703-2716. doi:10.1093/jxb/erx134

Filgueira, F.A.R. 2000. Novo manual de olericultura: Agrotecnologia moderna na produção e comercialização de hortaliças. Universidade Federal de Viçosa, Viçosa, BRA.

Finzi, R.R., G.M. Maciel, J.M.Q. Luz, A.A. Clemente, and A.C.S. Siquieroli. 2017. Growth habit in mini tomato hybrids from a dwarf line. Biosci. J. 33:52-56. doi:10.14393/BJ-v33n1a2017-35763

Haydar, A., M.A. Mandal, M.B. Ahmed, M.M. Hannan, R. Karim, M.A. Razvy, U.K. Roy, K. Akbar, I. Ullah, M. Shahid, A. Rehman, and M. Salahin. 2007. Studies on genetic variability and interrelationship among the different traits in tomato (Lycopersicon esculentum Mill.). Middle-East J. Sci. Res. 2(3-4):139-142.

Hefferon, K.L. 2016. Can biofortified crops help attain food security? Curr. Mol. Biol. Rep. 2:180-185. doi:10.1007/s40610016-0048-0

Holland, J.B., W.E. Nyquist, and C.T. Cervantes-Martínez. 2003. Estimating and interpreting heritability for plant breeding: an update. In: J. Janick, editor, Plant breeding reviews. Vol 22. John Wiley and Sons Inc., Hoboken, NJ, USA. p. 9-112. doi:10.1002/9780470650202.ch2

Khan, B.A., S.F. Mehboob, M. Ahmad, M. Iqbal, I. Ullah, M. Saleem, A. Rehman, and M. Shaid. 2017. Genetic analysis of F2 population of tomato for studying quantitative traits in the cross between Coldera x KHT5. Int. J. Plant Res. 7(4):90-93. doi:10.5923/j.plant.20170704.02

Kolota, E., and K. Adamczewska-Sowinska. 2001. Evaluation of new leek cultivars for early growing. Veg. Crops Res. Bull. 54:29-34.

Kumar, D., R. Kumar, S. Kumar, M.L. Bhardwaj, M.C. Thakur, R. Kumar, M.L. Bhardwai, M.C. Thakur, and P. Kumar. 2013. Genetic variability, correlation and path coefficient analysis in tomato. Int. J. Veg. Sci. 19:313-323. doi:10.1080/193152 60.2012 .726701

López, E., J. Gabriel, A. Angulo, J. Magne, J. La Torre, y M. Crespo. 2015. Herencia y relación genética asociados al rendimiento, madurez en híbridos de tomate [Solanum lycopersicum L.(Mill.)]. Agron. Costarricense 39(1):107-109.

Martínez, V., M. Nieves-Cordones, M. Lopez-Delacalle, R. Rodenas, T.C. Mestre, F. Garcia-Sanchez, and R.M. Rivero. 2018. Tolerance to stress combination in tomato plants: New insights in the protective role of melatonin. Molecules 23(3):E535. doi:10.3390/molecules23030535

Mérida, C., M. Colque, y H. Mercado. 2017. Evaluación agronómica de 116 híbridos experimentales de tomate (F1) desarrollados por el INIAF, en el Instituto de Investigaciones Agrícolas El Vallecito, Santa Cruz. Rev. Invest. Agropecu. For. Bol. 1(3):16-24.

Mohamed, S.M., E.E. Ali, and T.Y. Mohamed. 2012. Study of heritability and genetic variability among different plant and fruit characters of tomato (Solanum lycopersicum L.). Int. J. Sci. Technol. Res. 1(2):55-58. 
Nuez, V.F. 1995. El Cultivo del tomate. Mundi- Prensa, Madrid, ESP.

Peralta, G., J.C. Carrillo-Rodríguez, J.L. Chávez-Servia, A.M. Vera-Guzmán, e I. Pérez-León. 2012. Variación de caracteres agronómicos y licopeno en líneas avanzadas de tomate (Solanum lycopersicum L.). Phyton 81(1):15-22.

Pnueli, L., L. Carmel-Goren, D. Hareven, T. Gutfinger, J. Alvarez, M. Ganal, D. Zamir, and E. Lifschitz. 1998. The SELFPRUNING gene of tomato regulates vegetative to reproductive switching of sympodial meristems and is the ortholog of CEN and TFL1. Development 125:1979-1989.

Ramzan, A., T.N. Khan, N.N. Nawab, A. Hina, T. Noor, and G. Jillani. 2014. Estimation of genetic components in f 1 hybrids and their parents in determinate tomato (Solanum lycopersicum L.). J. Agric. Res. 52:65-75.

Resende, M.D.V. 2002. Genética biométrica e estatística no melhoramento de plantas perenes. Embrapa Florestas, Brasilia, BRA.

Resende, M.D.V. 2007. SELEGEN REML/BLUP: sistema estatístico e seleção genética computadorizada via modelos lineares mistos. Embrapa Florestas, Brasilia, BRA.

Resende, M.D.V. 2016. Software Selegen-REML/BLUP: a useful tool for plant breeding. Crop Breed. Appl. Biotechnol. 16:330339. doi:10.1590/1984-70332016v16n4a49

Saleem, M.Y., K.P. Akhtar, Q. Iqbal, M. Asghar, A. Hameed, and M. Shoaib. 2016. Development of tomato hybrids with multiple disease tolerance. Pak. J. Bot. 48:771-778.

Sharanappa, K.P., and S.C. Mogali. 2014. Studies on genetic variability, heritability and genetic advance for yield and yield components in F2 segregating population of tomato (Solanum lycopersicum L.). Karnataka J. Agric. Sci. 27:524-525. doi:10.18782/2320-7051.6097

Shokat, S., F.M. Azhar, G. Nabi, and Q. Iqbal. 2015. Estimation of heritability and genetic advance for some characters related to earliness in tomato (Solanum lycopersicum L.). J. Agric. Res. 53:351-356.

Vallejo, F.A. 1994. Estudios genéticos básicos para la creación de nuevos cultivares de tomate Lycopersicon esculentum Mill, adaptados a las condiciones de Colombia. Acta Agron. 44(1-4):9-168.

Vallejo, F.A. 1999. Mejoramiento genético y producción de tomate en Colombia. Universidad Nacional de Colombia, Cali, COL.

Vallejo, F.A., y M. Lobo. 1994. Heredabilidad del rendimiento y sus componentes en tomate, Lycopersicon esculentum, Mill; correlaciones genéticas y ambientales. Acta Agron. 44(1-4):85-94.

Wray, N., and P. Visscher. 2008. Estimating trait heritability. Nature Educ. 1(1):29.

Yelle, S., R.T. Chetelat, M. Dorais, J.W. Deverna, and A.B. Bennett. 1991. Sink metabolism in tomato fruit: IV. Genetic and biochemical analysis of sucrose accumulation. Plant Physiol. 95:1026-1035. doi:10.1104/pp.95.4.1026 